\title{
Museos de mujeres y eWOM: \\ Análisis y valoración
}

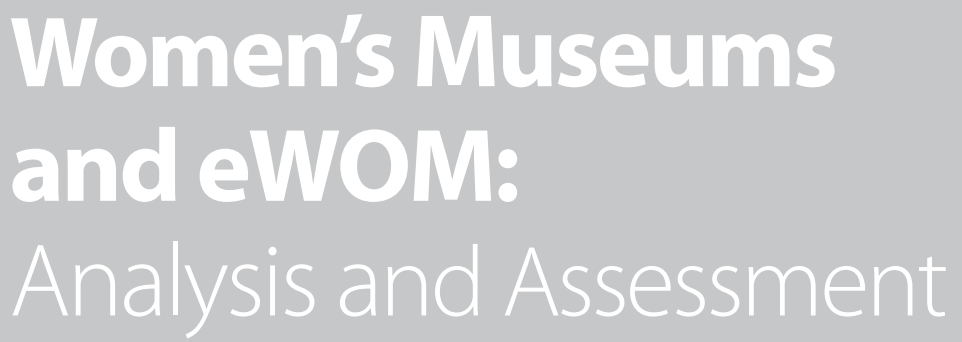

\section{Ruth Fernández-Hernández}

Doctoranda Universidad Rey Juan Carlos y profesora de ESIC Business \& Marketing School ruth.fernandez@esic.edu ORCID: https://orcid.org/0000-0001-6759-1843

\section{Dra. Natividad Crespo-Tejero}

Profesora de ESIC Business \& Marketing School

natividad.crespo@esic.edu

ORCID: https://orcid.org/0000-0003-4541-8120
Fernández-Hernández, R. y Crespo-Tejero, N. (2020) Museos de mujeres y eWOM: análisis y valoración

Revista Internacional de Investigación en Comunicación aDResearch ESIC. № 22 Vol 22

Monográfico especial, marzo 2020 · Págs. 98 a 117 https://doi.org/10.7263/adresic-022-05 
RESUMEN

\section{Clasificación JEL:}

M3, L86, Z32

Palabras clave:

«De boca en boca» electrónico (eWOM), museo,

Museo de mujeres, comunicación online, comunicación cultural
Objetivo del estudio: El presente estudio tiene como objetivo analizar los contenidos generados en el «de boca en boca» electrónico (eWOM) en una muestra de museos de mujeres internacionales, prestando atención a las evaluaciones recibidas por parte de los visitantes que puedan repercutir en la reputación online de los museos de mujeres.

Diseño/metodología/enfoque: Para alcanzar el objetivo se ha realizado un análisis de las opiniones, valoraciones y comentarios de los usuarios de la comunidad virtual TripAdvisor que han visitado los museos de mujeres de la muestra del estudio.

Resultados: Los resultados revelan que existe poca presencia de los museos de mujeres en la comunidad virtual analizada, sin embargo, los resultados de la comunicación eWOM son positivos, generando una buena reputación online de las instituciones.

Limitaciones/implicaciones: El estudio se centra exclusivamente en la plataforma TripAdvisor, pero los resultados muestran la relevancia del eWOM en la reputación de los museos para atraer visitantes al espacio físico.

Originalidad/contribución: Como consecuencia de los cambios sociales actuales y la relevancia del papel de la mujer en la sociedad, junto con el gran desarrollo de internet, este artículo demuestra la importancia que tiene para los museos de mujeres estar presentes en comunidades virtuales que faciliten su visibilidad y reputación online, y con ello, fomente el atractivo para los visitantes.

\section{ABSTRACT}

\section{JEL Classification: \\ M3, L86, Z32}

Key words:

"Electronic word of mouth" (eWOM), museum, Women's museum, online communication, cultural communication
Purpose: This study aims to analyse the content generated by electronic «word of mouth» (eWOM) in a sample of international women's museums, paying attention to the evaluations received from visitors that may have an impact on the online reputation of women's museums.

Design / Methodology / Approach: To achieve this objective, an analysis was made of the ratings, scores and comments of the users of the TripAdvisor virtual community who visited the women's museums in the study sample.

Results: The results reveal that there is little presence of women's museums in the virtual community analyzed, however, the results of eWOM communication are positive, generating a good online reputation of the institutions.

Limitations / Implications: The study focuses exclusively on the TripAdvisor platform, but the results show the relevance of eWOM on the reputation of museums to attract visitors to physical space.

Originality / Contribution: Given the current social changes and the relevance of the role of women in society, together with the great development of the Internet, this article demonstrates the importance for women's museums to be present in virtual communities that facilitate their visibility and reputation, and thus promote their attractiveness to visitors. 


\section{Introducción}

Tradicionalmente el rol de la mujer ha estado en un segundo plano en la sociedad. En los últimos años, se ha promovido por parte de instituciones públicas su papel en los diferentes ámbitos de la sociedad. En este contexto, los museos de mujeres han ido surgiendo paulatinamente y en paralelo con la evolución social, dando un papel más destacado a la mujer en el ámbito cultural.

Actualmente, los museos de mujeres representan una muestra minoritaria dentro del conjunto de museos globales, siendo fundamental para su desarrollo darse a conocer a través de los medios de comunicación empleados por la sociedad actual, como es el caso de internet y los medios sociales que proporciona. En el mundo virtual, el «de boca en boca» electrónico ('electronic word of mouth', eWOM) generado por el consumidor en los medios sociales ha ido adquiriendo credibilidad en los usuarios hasta convertirse en una importante herramienta de información para los visitantes de los museos (Hausmann, 2012). Sin embargo, el análisis del eWOM en las instituciones museísticas es aún escaso, por ese motivo se ha considerado la importancia de analizar los contenidos generados por los usuarios a través de eWOM, debido al impacto que puede tener para los museos de mujeres en la reputación online y en la capacidad de atraer nuevos visitantes.

El trabajo presentado se estructura en las siguientes partes: en primer lugar se presenta una revisión breve de la literatura sobre los museos de mujeres y el «de boca en boca» electrónico (eWOM). Posteriormente se describen los objetivos de la investigación y la metodología empleada. A continuación, se muestran los resultados descriptivos del análisis realizado sobre los contenidos de eWOM en los museos seleccionados, y finalmente se exponen las principales conclusiones obtenidas del estudio.

\section{Marco teórico}

\subsection{Museos de mujeres}

Las mujeres han reclamado su espacio desde el siglo XIX, en 1948 Elizabeth Cady Stanton y Lucretia Mott congregan a cientos de personas en la primera convención nacional por los derechos de las mujeres en Nueva York donde exigen derechos civiles, sociales, políticos y religiosos basándose en que los hombres y las mujeres son creados iguales (ONU Mujeres, 2019). A partir de aquí comienza un movimiento que perdura en nuestros días y que ha pasado por diferentes hitos como la demanda del sufragio femenino en 1893, la celebración del primer Día Internacional de la Mujer en 1911, la incorporación de la mujer al ámbito laboral durante la I y II Guerra Mundial. En 1945 nace Naciones Unidas y en 1946 la Comisión de la Condición Jurídica y Social de la Mujer se convierte en el primer órgano intergubernamental mundial dedicado exclusivamente a la igualdad de género. En la Declaración de los Derechos Humanos, aprobada por la Asamblea General de las Naciones Unidas en 1948 y que en 2018 ha cumplido 70 años, se enunciaron los derechos civiles, políticos, económicos, sociales y culturales elementales que todos los seres humanos (tanto hombres como mujeres) deben disfrutar (ONU, 2019). La Declaración de los Derechos de la Mujer (en sus siglas en inglés CEDAW) aprobada en 1979 exige legalmente a los gobiernos eliminar todas las formas de discriminación contra las mujeres en la esfera pública y privada, con el objetivo de alcanzar la igualdad entre hombres y mujeres. A finales del siglo XX se firman tratados históricos como la Declaración sobre la Eliminación de la Violencia contra las mujeres en 1993, el Programa de la acción de la CIPD en 1994, la Declaración y Plataforma de Acción de Beijing en 1995, la Resolución 1325 del Consejo de Seguridad en 2000 y la Declaración del Milenio de las Naciones 
Unidas en 2000 donde se fijaron ocho objetivos para lograr el fin de la pobreza, revisándose en 2015 y fijando los Objetivos de Desarrollo Sostenible (ODS). En 2010, ONU Mujeres se convierte en el primer organismo de las Naciones Unidas en trabajar exclusivamente por los derechos de la mujer. Desde 2011 se están produciendo movimientos de mujeres en los Estados Árabes. En 2013 Malala Yousafzai da un discurso en público en Naciones Unidas tras sobrevivir a un ataque por ser una activista por la educación en Pakistán. En 2017 entre 3,5 y 5,5 millones de personas asisten a la «marcha de las mujeres» por los derechos de la mujer. El siglo XXI se caracteriza por el activismo digital, los hashtags \#NiUnaMenos, \#MeToo, \#YoTambien..., (ONU Mujeres, 2019) son conocidos en todo el mundo.

La comunicación ha sido fundamental en el conocimiento y transmisión de estos hechos desde la aparición de la imprenta, la radio, la televisión y el teléfono hasta la actualidad con el desarrollo de internet, que ha proporcionado a las mujeres una manera de expresarse sin las limitaciones del ámbito físico (Galarza, CastroMartinez y Sosa, 2019), en cuanto a los medios, el tiempo y el espacio. Internet ha proporcionado un espacio que permite la participación en diferentes ámbitos, permitiendo el desarrollo de acciones colectivas y movimientos sociales con el objetivo de conseguir el cambio social (Hwang y Kim, 2015). Los movimientos sociales promovidos por las mujeres han afectado directamente al sector cultural y en consecuencia a los museos. El Consejo Internacional de Museos (ICOM, 2019) lo definió como: «una institución permanente, sin fines de lucro, al servicio de la sociedad y de su desarrollo, abierta al público que adquiere, conserva, estudia, expone y transmite el patrimonio material e inmaterial de la humanidad y de su medio ambiente con fines de educación y delei- te». Siguiendo esta definición los museos no han quedado al margen de los movimientos sociales de las mujeres que se han producido, siendo en los años 50 cuando se crean los primeros museos en Estados Unidos y Australia teniendo como tema central la mujer, posteriormente en los años 80 empiezan a crearse en Europa (Schönweger, 2010). En España el primer museo de la mujer que abrió sus puertas fue en 2006 el Museo Etnográfico de la Mujer Gitana en Granada (Cáceres, 2010). El concepto de museo de la mujer recoge dos conceptos, por un lado, el de mujer que indica la temática, por otro lado, el museo que es la institución que recoge el conocimiento sobre el tema (Schönweger, 2010), su objetivo es difundir el patrimonio femenino y la participación que las mujeres han tenido en el desarrollo de la economía, la sociedad, la política y la cultura en sus países o a nivel internacional, en el pasado y en la actualidad para superar la discriminación de género y contribuir a una sociedad más igualitaria (De Carvalho y Vaquinhas, 2015).

En los últimos años se han desarrollado numerosos museos de la mujer, el Ministerio de Cultura y Deporte de España (MCD) en su web tiene registrados cuarenta y ocho museos de veintinueve países a nivel internacional, cuatro españoles, cinco latinoamericanos y treinta y nueve en el resto del mundo. Además, se están poniendo en marcha diferentes proyectos (Benito y Molina, 2010) un ejemplo de ello es la creación en 2008 de la plataforma virtual Women in Museum, fue también en ese año cuando se celebró el primer Congreso Internacional de Museos de mujeres en Italia y que en la actualidad sigue celebrándose (Benito y Molina, 2010). En el año 2012 surge la Asociación Internacional de Museos de la Mujer (International Association of Women's Museums, IAWM) fundada en Australia, con los objetivos de fomentar el intercambio, el apoyo y cooperación 
entre los museos de mujeres en todo el mundo y promover la cultura, las artes, la educación y la formación desde una perspectiva de género. Otras iniciativas han sido las exposiciones dedicadas a las mujeres en grandes museos como la celebrada en el Centro Pompidou que desde 2009 muestra la exposición «elles@centrepompidou»dedicada a las artistas femeninas reuniendo obras de artistas femeninas desde principios del siglo XX hasta nuestros días. El Museo Nacional Centro de Arte Reina Sofía entre abril y septiembre de 2018 con la muestra «Dora García. Segunda Vez» o la del Museo del Prado «Historia de dos pintoras: Sofonisba Anguissola y Lavinia Fontana» desde octubre 2019 a febrero 2020. Desde el punto de vista académico cada vez más la mujer está siendo un tema que despierta el interés en las investigaciones centradas en el ámbito de la museología, no sólo por su presencia en las obras de arte o por el cada vez más creciente número de artistas mujeres, sino también por su forma de comunicarse, opinar y valorar a través de las herramientas que tienen a su disposición.

Internet ha supuesto un refuerzo muy importante para uno de los cometidos de los museos, ser relevante para la sociedad (Del Río-Castro, 2011) y fomentar una de sus funciones, la difusión. La importante aportación de Internet a los museos radica en la influencia de la comunicación eWOM. Medios sociales, como TripAdvisor, ampliamente estudiados por diversos investigadores (Banerjee y Chua, 2016; Ferguson, Piché y Walby, 2015; Pearce y Wu, 2015; Xie, Zhang y Zhang, 2014) y responsables de comunicación, demuestran la influencia que tienen en la toma de decisiones de los turistas a nivel internacional. Los consumidores califican, puntúan, pero sobre todo transmiten sus experiencias y sentimientos que es lo que motiva a otros consumidores, ya que con la experiencia de estos minimizan sus riesgos en la toma de decisiones (Kim y Park, 2017). En este sentido las comunidades virtuales, como TripAdvisor adquieren una gran relevancia en la reputación online corporativa de las organizaciones.

La investigación sobre eWOM en la industria del turismo ha aumentado de modo considerable en los últimos años, aunque son escasos los estudios que analizan eWOM en el consumo cultural. Este hecho sorprende ya que el eWOM muestra las respuestas de comportamiento de los visitantes respecto a su satisfacción o insatisfacción con su experiencia en la visita física a un museo. Además, el conocimiento de esta información puede ayudar a los museos a mejorar la experiencia de los públicos ofreciéndoles exposiciones y actividades adecuadas a sus demandas, mejores servicios e instalaciones más accesibles y cómodas (Kotler y Kotler, 2000) con el fin de mejorar la relación con sus visitantes y por consiguiente su imagen corporativa. De hecho, existe una tendencia creciente de eWOM sobre museos en las plataformas de revisión más populares, siendo las referencias personales una de las principales razones para hacer una visita al museo, dadas las características intrínsecamente experimentales del consumo cultural (Hausmann, 2012).

En definitiva, la comunicación eWOM utiliza la naturaleza efímera, anónima y a gran escala de Internet e introduce una nueva forma de capturar, analizar e interpretar la influencia de la comunicación en el turismo (Yoo, Lee y Lee, 2015; Filieri y McLeay, 2014).

\subsection{Electronic word of mouth (eWOM)}

Los medios sociales han posibilitado la generación de contenidos por parte de los usuarios y la comunicación entre los consumidores facilitando la aparición de una nueva forma de «de boca en boca», denominado «de boca en boca» electró- 
nico ('electronic word of mouth', eWOM). Según Hennig-Thurau et al., (2004, p.39) eWOM se define como «cualquier declaración positiva o negativa hecha por clientes potenciales, actuales o anteriores sobre un producto o empresa, que se pone a disposición de una multitud de personas e instituciones a través de Internet».

Existe una fuerte evidencia empírica de que el WOM tradicional influye tanto en las actitudes de los consumidores (Engel, Kegerreis y Blackwell, 1969) como en el comportamiento (Arndt, 1967; Katz y Lazarsfeld, 1955). El «de boca en boca» tradicional se desarrolla a través de conversaciones verbales, en un contexto cara a cara, simultáneas y bidireccionales con amigos y/o familiares o proveedores que ocurren de manera momentánea. Sin embargo, el «de boca en boca» generado a través de los medios sociales transmite opiniones a través de la palabra escrita, normalmente se produce entre desconocidos y los contenidos en Internet se mantienen durante mucho tiempo. De esta manera, muchos consumidores pueden leer el contenido y decidir si responden o no a la fuente (Bickart y Schindler, 2001; López y Sicilia, 2014). Esto tiene implicaciones importantes; por un lado, el eWOM es persistente y la información está disponible «bajo demanda» para otros individuos que analizan valoraciones sobre productos y servicios (Hennig-Thurau et al., 2010). Por otro lado, la persistencia y la observabilidad facilitan que el eWOM existente, tenga un impacto relevante en el eWOM futuro (Bowman y Narayandas, 2001). En este sentido, el eWOM puede ser considerado como endógeno, ya que está influenciado por las ventas del producto o servicio (Godes y Mayzlin, 2004). Al generar contenidos sobre las experiencias de los productos o servicios adquiridos, el eWOM puede afectar al comportamiento futuro, además del comportamiento actual. Otro aspecto a destacar es el anonimato; que permite a los consumidores participar en foros de opinión sin mostrar su identidad favoreciendo el poder expresarse con más libertad que en una situación cara a cara y evitando las respuestas de deseabilidad social (Goldsmith y Horowitz, 2006).

Los usuarios a través de los nuevos medios de comunicación se han convertido en individuos muy activos con extensa información sobre productos, marcas y servicios. De este modo, asumen un rol de clientes, pero también un papel de productores de contenidos, estando fuertemente interconectados entre ellos mediante las comunidades virtuales. En este contexto, el eWOM se presenta como un dispositivo de comunicación poderoso y eficaz, donde los usuarios pueden acceder a él desde diferentes lugares a través de Internet (Bakos y Dellarocas, 2011) y facilita a distintos individuos transmitir sus valoraciones simultáneamente en el mismo sitio web (Lee, Park y Han, 2008). Complementariamente, el uso de las comunicaciones eWOM durante el proceso de decisión de compra reduce el tiempo en la toma de decisiones, minimiza el riesgo de tomar malas decisiones de compra, e implica que los consumidores perciban mayor seguridad a la hora de comprender los productos/servicios (HennigThurau y Walsh, 2003).

El contenido de eWOM puede incluir evaluaciones positivas mostrando descripciones agradables, atractivas y atrayentes de productos o servicios, mientras que las comunicaciones negativas de eWOM suelen incluir comentarios y descripciones negativas y desagradables (Robinson y Valor, 2013). En este sentido, la imagen y reputación de las organizaciones puede verse interferida por los contenidos generados por los usuarios, incidiendo sobre los mensajes de marketing tradicionales de las empresas (HennigThurau et al., 2010). Los comentarios positivos 
de eWOM favorecen la calidad esperada hacia ese producto o servicio y generan actitudes positivas, mientras que los comentarios negativos se basan en las experiencias insatisfechas hacia un producto o servicio, que genera comentarios de quejas por parte de los consumidores (Silverman, 2001). En general, pueden implicar descrédito del producto o servicio y tienen un impacto desfavorable en las actitudes hacia el producto (Liu, 2006). Por otro lado, el volumen de eWOM se refiere a la cantidad total de interacciones. Según (Liu, 2006, p. 77) «cuanto mayor sea el volumen de eWOM, mayor será la probabilidad de que la información llegue a oídos del consumidor». Además, el volumen de eWOM puede incrementar el conocimiento de los usuarios sobre el servicio/ producto y reducir su indecisión sobre la elección de compra (Chen, Wang y Xie, 2011).

La comunicación eWOM ha sido analizada en función de las características de la fuente, el mensaje y el receptor (Ismagilova et al., 2019). Por un lado, la experiencia y la confiabilidad de la fuente son factores significativos para aumentar la persuasión de las comunicaciones eWOM e incrementar la intención de compra (Saleem y Ellahi, 2017). Por otro lado, la influencia del mensaje de eWOM viene determinado por factores como la calidad del argumento (Tsao y Hsieh, 2015), la credibilidad (Koo, 2016) o la confianza del mensaje (Ho y Chang, 2010). Por último, el receptor mostrará una predisposición positiva hacia la comunicación eWOM, si existe previamente una actitud positiva hacia las compras en línea (Hsu, Lin y Chiang, 2013) y hacia el sitio web (Lee et al., 2011).

Complementariamente, el impacto de eWOM en los consumidores y en las organizaciones ha sido objeto de estudio por los investigadores en los últimos años. El análisis realizado se ha centrado en investigar el efecto de eWOM sobre las actitudes de los consumidores (Ahluwalia, 2002; Doh y Hwang, 2009), intención de compra (East et al., 2017; Ismagilova et al., 2019), volumen de ventas (Floyd et al., 2014; Babić et al., 2015; You, Vadakkepatt y Joshi, 2015) y resultados financieros de las empresas (Liu, 2019; Xun y Guo, 2017).

Otro elemento analizado ha sido el impacto de eWOM en la reputación de las organizaciones. La reputación corporativa implica un juicio global de los consumidores sobre los atributos de una organización, que puede cambiar con el tiempo y que se ve modificada a partir de la información recibida (Balmer y Gray, 2003). En términos generales, la buena reputación corporativa aumenta la probabilidad de que los usuarios se involucren en una comunicación eWOM positiva sobre la organización (Hidayat et al., 2017; Hong y Yang, 2009) y disminuye la intención de generar eWOM negativo (Duygun, Menteş y Kubaş, 2014). Sin embargo, las comunicaciones virales a través de plataformas que faciliten un eWOM negativo tienen relevantes consecuencias negativas para la reputación corporativa de las organizaciones (Williams, Buttle y Biggemann, 2012). Además, el efecto del eWOM es mayor para los sitios web con reputación establecida (Park y Lee, 2009). En esta línea, Reyes-Menendez, Saura y Martinez-Navalon (2019) entienden que la credibilidad influye en la reputación de las empresas en el sector turístico que tienen un perfil en plataformas online, como TripAdvisor. Este aspecto podría afectar a la reputación online de las empresas, por ejemplo, del sector turístico.

En definitiva, el impacto del eWOM positivo puede fomentar el efecto en una doble vía: por un lado, los consumidores de marcas con una buena reputación estarán más implicados para establecer comunicaciones eWOM positivas en las plataformas digitales y, por otro lado, la pro- 
pia comunicación eWOM tenderá a robustecer la reputación de la marca. Sin embargo, el efecto del comportamiento de descontento de los consumidores provocado por sentimientos negativos de insatisfacción, injusticia o ira se considera un objetivo a tener en cuenta para proteger la reputación de las organizaciones.

Las comunidades virtuales son sitios eWOM en las cuales los usuarios interesados en una misma temática interactúan para tener información e interaccionar (Cothrel, 2000). TripAdvisor es una comunidad virtual de viajes, un sitio de opiniones sobre hoteles, restaurantes y lugares culturales, donde los usuarios comparten información de los lugares visitados, en formato texto y visual, además de valorarlos y puntuarlos (TripAdvisor, 2019). Es un referente mundial para las personas a la hora de planificar su viaje en cuanto a la decisión de qué hotel reservar, a qué restaurante acudir, cómo organizar su tiempo libre y en consecuencia qué visitar (Jeacle y Carter, 2011; Gavilan, Martínez-Navarro y FernándezLores, 2017). Desde hace tiempo, TripAdvisor publica anualmente los Travellers Choice de los mejores museos del mundo y los Travellers Choice de España, son ranking de los que se hacen eco gran cantidad de medios de comunicación, tanto online como offline, siendo un referente para muchos usuarios ya que les proporciona vivencias, experiencias y satisfacciones de otras personas que los han visitado (Ferguson, Piche y Walby, 2015), mediante las fotos y comentarios personales que publican, siendo estos elementos de persuasión que influirán en el comportamiento de los consumidores (Zhang, Zhang y Yang, 2016; Sparks, Perkins y Buckley, 2013). Los usuarios de TripAdvisor son evaluadores de los productos turísticos, como los museos, pero también se convierten en activistas en línea o críticos sociales (Mkono y Tribe, 2016).
De la revisión de la literatura podemos concluir que el contenido de eWOM generado a través de comunidades virtuales tiene cada vez mayor peso en la reputación de las organizaciones. En base a esta premisa, y dada la escasez de estudios sobre los contenidos generados en comunidades virtuales en el ámbito museístico, nuestro artículo plantea como principal objetivo analizar los contenidos generados en el eWOM en una muestra de museos de mujeres internacionales, prestando atención a las valoraciones recibidas por parte de los visitantes que puedan repercutir en la reputación online de los museos de mujeres.

\section{Metodología}

La metodología llevada cabo en esta investigación es de carácter cuantitativo y cualitativo, con un diseño no experimental de carácter exploratorio y descriptivo. La muestra de museos de mujeres analizados en el estudio, se ha seleccionado a partir de la página web del Ministerio de Cultura y Deporte de España. La muestra inicial está formada por cuarenta y ocho museos de veintinueve países, de los cuales cuatro son españoles, cinco latinoamericanos y treinta y nueve del resto del mundo. Después de realizar un análisis para determinar los museos que están presentes en la comunidad virtual TripAdvisor, la muestra de museos se reduce a veinte de ellos, que son los que se muestran en la Tabla 1, y sobre los que se ha realizado el análisis.

La elección de la comunidad virtual TripAdvisor para el presente estudio queda justificada por el elevado volumen de opiniones y comentarios que se generaron en ella (en 2019, más de 830 millones de opiniones y comentarios de 8,6 millones de alojamientos, restaurantes, experiencias, vuelos y cruceros). Además, es una comunidad positivamente considerada, que permite a sus usuarios compartir videos, fotos y mantener 


\section{Tabla 1. Museos de mujeres analizados}

\begin{tabular}{|c|c|}
\hline País & Museos de Mujeres \\
\hline \multirow{2}{*}{ Alemania } & Frauenmuseum Bonn \\
\hline & Käthe Kollwitz Museum \\
\hline Australia & National Pioneer Women's Hall of Fame \\
\hline Austria & Frauenmuseum \\
\hline Corea & Women's History Exhibition Hall \\
\hline Costa de Marfil & Musée des civilisations de Côte d'Ivoire, Women's Museum initiative \\
\hline Dinamarca & Kvindemuseet i Denmark \\
\hline \multirow{3}{*}{ España } & Casa museo Emilia Pardo Bazán \\
\hline & Casa museo Rosalía de Castro \\
\hline & Museo etnológico de la mujer gitana \\
\hline \multirow{4}{*}{ Estados Unidos } & International Museum of Women \\
\hline & National Museum of Women in the Arts \\
\hline & National Women's. Hall of Fame \\
\hline & National Women's History Museum \\
\hline \multirow{2}{*}{ México } & Museo de Frida Kahlo \\
\hline & Museo de mujeres artistas mexicanas \\
\hline Noruega & Kvinnemuseet \\
\hline Suecia & Museum Anna Nordlander \\
\hline \multirow{2}{*}{ Vietnam } & The South Vietnam Women's Museum \\
\hline & Vietnam Women Museum \\
\hline
\end{tabular}

conversaciones con otros usuarios. Está presente en 49 mercados, 28 idiomas y presenta alrededor de 460 millones de visitantes únicos al mes (TripAdvisor, 2019).

Para conseguir los objetivos marcados, se han recogido datos a partir de la comunidad virtual TripAdvisor de las siguientes variables:

- Opiniones: el sistema de valoración de TripAdvisor permite valorar las experiencias de los usuarios en una escala de 1 a 5 puntos (excelente, muy bueno, normal, malo, pésimo).
- Valoraciones: resumen de las puntuaciones que los usuarios conceden en sus valoraciones globales según la experiencia que hayan tenido. TripAdvisor denomina a esta valoración «puntuación de burbujas». La puntuación se muestra en una escala de 1 a 5; donde 3 equivale a una puntuación media y el 5 a una puntuación excelente (TripAdvisor, 2019).

- Número de comentarios de los visitantes.

- Contenidos generados por los usuarios: contenidos sobre las experiencias, vivencias, in- 
formación recibida, accesibilidad, trato recibido, etc. A partir de los contenidos analizados se han utilizado «palabras clave» para clasificar las opiniones de los usuarios en opiniones positivas, negativas y neutras. Por ejemplo, «palabras clave» para identificar opiniones positivas (maravilloso, impresionante, fabuloso), opiniones negativas (difícil, carente, malo) y opiniones neutras (descripciones generales, por ejemplo: «tiene tienda»).

El proceso de recogida de datos se ha desarrollado durante los meses de noviembre y diciembre del año 2019.

\section{Resultados y conclusiones}

En este apartado se incluyen los principales resultados obtenidos en la investigación. En primer lugar, se presentan los datos sobre los porcentajes de opiniones de los visitantes reflejados en una escala de 1 a 5 , donde 1 es pésimo y 5 excelente (Tabla 2). Los datos mostrados reflejan que la valoración de las experiencias de los visitantes a los museos analizados, se encuentran mayoritariamente, en la escala de excelente y muy bueno. Lo que refleja que la experiencia del visitante es en general satisfactoria.

\section{Tabla 2. Valoración, en porcentaje, opiniones de los visitantes}

\begin{tabular}{|c|c|c|c|c|c|}
\hline Museos de Mujeres & $\begin{array}{c}5 \\
\text { Excelente }\end{array}$ & $\begin{array}{c}4 \\
\text { Bueno }\end{array}$ & $\begin{array}{c}3 \\
\text { Normal }\end{array}$ & $\begin{array}{c}2 \\
\text { Malo }\end{array}$ & $\begin{array}{c}1 \\
\text { Pésimo }\end{array}$ \\
\hline Frauenmuseum Bonn & $40 \%$ & $30 \%$ & $30 \%$ & $0 \%$ & $0 \%$ \\
\hline Käthe Kollwitz Museum & $66 \%$ & $27 \%$ & $6 \%$ & $0 \%$ & $1 \%$ \\
\hline National Pioneer Women_s Hall of Fame & $50 \%$ & $41 \%$ & $7 \%$ & $1 \%$ & $1 \%$ \\
\hline Frauenmuseum & $60 \%$ & $26 \%$ & $8 \%$ & $0 \%$ & $6 \%$ \\
\hline Women's History Exhibition Hall & $20 \%$ & $40 \%$ & $40 \%$ & $0 \%$ & $0 \%$ \\
\hline Musée des civilisations de Côte d'Ivoire, Women's Museum initiative & $20 \%$ & $36 \%$ & $24 \%$ & $12 \%$ & $8 \%$ \\
\hline Kvindemuseet i Denmark & $41 \%$ & $36 \%$ & $16 \%$ & $4 \%$ & $3 \%$ \\
\hline Casa museo Emilia Pardo Bazán & $51 \%$ & $45 \%$ & $2 \%$ & $0 \%$ & $2 \%$ \\
\hline Casa museo Rosalía de Castro & $36 \%$ & $41 \%$ & $17 \%$ & $3 \%$ & $3 \%$ \\
\hline Museo etnológico de la mujer gitana, Barrio Sacramonte, Granada & $0 \%$ & $67 \%$ & $33 \%$ & $0 \%$ & $0 \%$ \\
\hline International Museum of Women & $90 \%$ & $10 \%$ & $0 \%$ & $0 \%$ & $0 \%$ \\
\hline National Museum of Women in the Arts & $62 \%$ & $25 \%$ & $8 \%$ & $3 \%$ & $2 \%$ \\
\hline National Women's. Hall of Fame & $49 \%$ & $29 \%$ & $17 \%$ & $4 \%$ & $1 \%$ \\
\hline National Women's History Museum & $0 \%$ & $0 \%$ & $0 \%$ & $0 \%$ & $0 \%$ \\
\hline Museo de Frida Kahlo & $63 \%$ & $25 \%$ & $9 \%$ & $2 \%$ & $1 \%$ \\
\hline Museo de mujeres artistas mexicanas & $81 \%$ & $15 \%$ & $2 \%$ & $1 \%$ & $1 \%$ \\
\hline Kvinnemuseet & $43 \%$ & $43 \%$ & $14 \%$ & $0 \%$ & $0 \%$ \\
\hline Museum Anna Nordlander & $0 \%$ & $0 \%$ & $0 \%$ & $0 \%$ & $0 \%$ \\
\hline The South Vietnam Women's Museum & $16 \%$ & $51 \%$ & $22 \%$ & $8 \%$ & $3 \%$ \\
\hline Vietnam Women Museum & $58 \%$ & $32 \%$ & $8 \%$ & $1 \%$ & $1 \%$ \\
\hline
\end{tabular}


En la Tabla 3 se muestran los datos de las valoraciones de cada museo. Según TripAdvisor, este dato aporta información resumida de las puntuaciones que los usuarios conceden en sus valoraciones en función de la experiencia que hayan tenido.

De los 20 museos de mujeres analizados podemos señalar que las valoraciones que los visitantes les otorgan son positivas, con puntuaciones iguales o superiores a 4 en el $75 \%$ de los casos (Figura 1), siendo las valoraciones de excelente (5) y bueno (4) las más numerosas (Tabla 2). Este dato sugiere que la experiencia de los visitantes en la visita física al museo ha sido satisfactoria. Ninguno de los museos analizados presenta una puntuación inferior a 3 puntos en una escala de 1 a 5 puntos. Por último, señalar que dos de los casos analizados (National Women's History Museum y Museum Anna Nordlander) tienen presencia en TripAdvisor, pero no tienen ninguna valoración ni comentarios.

La Tabla 4 muestra el número de opiniones que tiene cada uno de los museos analizados. Los resultados señalan que un $50 \%$ de los museos presentan una cantidad total de opiniones que

\section{Tabla 3. Valoración de cada museo}

\begin{tabular}{|c|c|}
\hline Museos de Mujeres & Valoración \\
\hline Frauenmuseum Bonn & 4 \\
\hline Käthe Kollwitz Museum & 4,5 \\
\hline National Pioneer Women_s Hall of Fame & 4,5 \\
\hline Frauenmuseum & 4,5 \\
\hline Women's History Exhibition Hall & 4 \\
\hline Musée des civilisations de Côte d'Ivoire, Women's Museum initiative & 3,5 \\
\hline Kvindemuseet i Denmark & 4 \\
\hline Casa museo Emilia Pardo Bazán & 4,5 \\
\hline Casa museo Rosalía de Castro & 4 \\
\hline Museo etnológico de la mujer gitana, Barrio Sacramonte, Granada & 3,5 \\
\hline International Museum of Women & 5 \\
\hline National Museum of Women in the Arts & 4,5 \\
\hline National Women's. Hall of Fame & 4 \\
\hline National Women's History Museum & 0 \\
\hline Museo de Frida Kahlo & 4,5 \\
\hline Museo de mujeres artistas mexicanas & 4,5 \\
\hline Kvinnemuseet & 4,5 \\
\hline Museum Anna Nordlander & 0 \\
\hline The South Vietnam Women's Museum & 3,5 \\
\hline Vietnam Women Museum & 4,5 \\
\hline
\end{tabular}




\section{Figura 1. Número de museos y su valoración en TripAdvisor}

Número de museos — Puntuación sobre 5

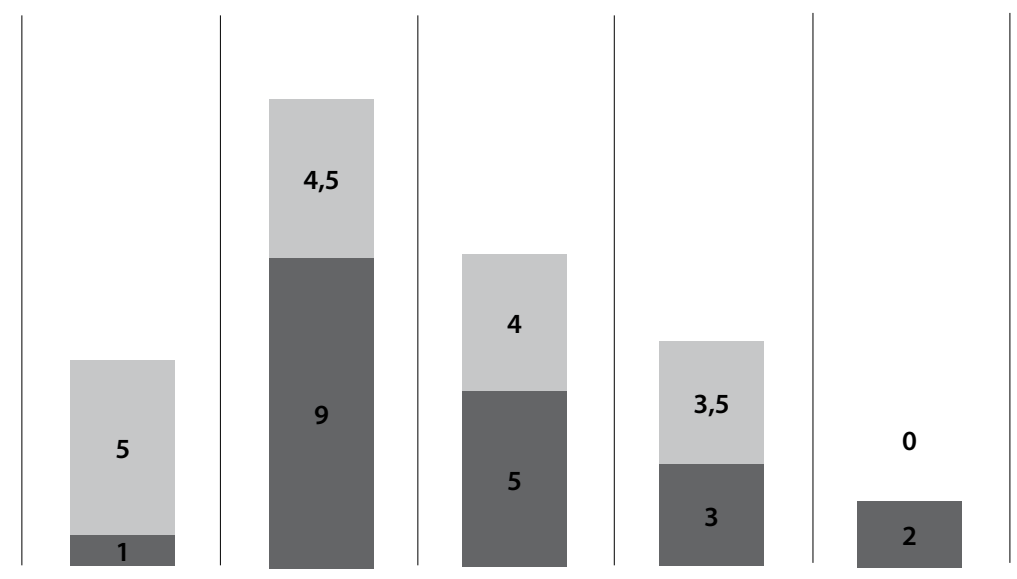

no supera los 50 comentarios. Este dato de escaso volumen de eWOM podría ser un freno en el conocimiento de los futuros visitantes sobre el museo y aumentar su indecisión sobre la elección del museo a visitar (Chen, Wang y Xie, 2011).
Por otro lado, cabe destacar que un $10 \%$ de los museos presentan un elevado volumen de comentarios, enfatizando el museo de Frida Kahlo en México, siendo muy superior el número de opiniones registradas frente al resto de museos analizados.

\section{Tabla 4. Número de opiniones en TripAdvisor}

\begin{tabular}{|l|l|c|}
\hline \multirow{2}{*}{ País } & Museos de Mujeres & Opiniones \\
\hline Alemania & Frauenmuseum Bonn & 10 \\
\hline & Käthe Kollwitz Museum & 225 \\
\hline Australia & National Pioneer Women_s Hall of Fame & 256 \\
\hline Austria & Frauenmuseum & 35 \\
\hline Corea & Women's History Exhibition Hall & 5 \\
\hline Costa de Marfil & Musée des civilisations de Côte d'Ivoire, Women's Museum initiative & 50 \\
\hline Dinamarca & Kvindemuseet i Denmark & 95 \\
\hline España & Casa museo Emilia Pardo Bazán & 47 \\
\hline
\end{tabular}




\section{Tabla 4. Número de opiniones en TripAdvisor (continuación)}

\begin{tabular}{|l|l|c|}
\hline \multirow{2}{*}{ País } & Museos de Mujeres & Opiniones \\
\hline \multirow{2}{*}{ España } & Casa museo Rosalía de Castro & 70 \\
\hline & Museo etnológico de la mujer gitana, Barrio Sacramonte, Granada & 3 \\
\hline Estados Unidos & International Museum of Women & 10 \\
\hline & National Museum of Women in the Arts & 360 \\
\hline México & National Women's. Hall of Fame & 106 \\
\hline Noruega & Museo de Frida Kahlo & 0 \\
\hline Suecia & Museo de mujeres artistas mexicanas & 9.469 \\
\hline Vietnam & Kvinnemuseet & 817 \\
\hline & Museum Anna Nordlander & 7 \\
\hline
\end{tabular}

En cuanto al análisis del contenido de las opiniones, en general destaca la referencia a las experiencias vividas durante la visita, el conocimiento descubierto y las emociones generadas. Se observa que las opiniones suelen manifestar evaluaciones positivas mostrando descripciones agradables del edificio y entorno del museo, de su organización y de los objetos que en él se muestran. Cabe destacar que en multitud de comentarios se hace referencia al rol de la mujer y su papel en la historia política, cultural y económica reflejando una muestra de la inquietud que el papel de la mujer despierta en la sociedad actual. Otro aspecto destacable es la referencia a servicios complementarios del museo como son la cafetería o la tienda, destacándose en los comentarios si es agradable o no comer en la cafetería o si la tienda tiene objetos de regalo interesantes. También se hace referencia al personal de la institución, en cuanto a la atención que brindan al visitante, las explicaciones que dan y los idiomas que hablan. Esto se refleja en la experiencia positiva cuando se sienten bien atendidos en cuanto al trato personal y si la comunicación se ha establecido en su propio idioma o en inglés, frente a otros idiomas más desconocidos o minoritarios. Por último, es destacable que en los comentarios se hace referencia a los dispositivos tecnológicos de los que dispone el museo, como son pantallas, audioguías, tarjetas interactivas, etc. Haciendo alusión a que estas herramientas han favorecido la experiencia enriqueciendo la información y guiando al visitante cuando han sido bien utilizadas o han funcionado de forma correcta, pero también cuando no han funcionado o no están bien planteadas dichas herramientas se refleja la experiencia negativa del visitante, generando comentarios negativos sobre este aspecto olvidándose de otros aspectos de la visita. 
El análisis de las dimensiones emocionales que despiertan los museos se presentan en la Tabla 5. Sobre la base de la revisión de la literatura, hemos catalogado las declaraciones generadas por los usuarios en función de «palabras clave», verificadas por un panel de profesionales, para identificar el tipo de valencia de eWOM. Como se describe en la metodología se han identificado aquellos comentarios que pueden considerarse positivos, negativos o neutros en cada museo analizado. Los resultados muestran que la gran mayoría de los museos (85 \%) tienen eWOM positivo; mientras que el porcentaje de museos con eWOM negativo es menor (65\%). Este dato se considera de gran interés, dado que recientes estudios confirman el impacto en las actitudes e intenciones de compra de la valencia positiva en las revisiones en línea (Tata, Prashar y Gupta, 2019).

\section{Tabla 5. Análisis de las dimensiones emocionales}

\begin{tabular}{|c|c|c|c|}
\hline \multirow{2}{*}{ Museos de Mujeres } & \multicolumn{3}{|c|}{ Sentimiento } \\
\hline & Positivo & Neutro & Negativo \\
\hline Frauenmuseum Bonn & $x$ & $x$ & $x$ \\
\hline Käthe Kollwitz Museum & $x$ & $x$ & \\
\hline National Pioneer Women_s Hall of Fame & $x$ & & $x$ \\
\hline Frauenmuseum & $x$ & & \\
\hline Women's History Exhibition Hall & $x$ & & $x$ \\
\hline Musée des civilisations de Côte d'Ivoire, Women's Museum initiative & $x$ & $x$ & $x$ \\
\hline Kvindemuseet i Denmark & $x$ & $x$ & $x$ \\
\hline Casa museo Emilia Pardo Bazán & $x$ & & $x$ \\
\hline Casa museo Rosalía de Castro & $x$ & & $x$ \\
\hline Museo etnológico de la mujer gitana, Barrio Sacramonte, Granada & $x$ & & \\
\hline International Museum of Women & $x$ & & \\
\hline National Museum of Women in the Arts & $x$ & & $x$ \\
\hline National Women's. Hall of Fame & $x$ & $x$ & $x$ \\
\hline \multicolumn{4}{|l|}{ National Women's History Museum } \\
\hline Museo de Frida Kahlo & $x$ & $x$ & $x$ \\
\hline \multicolumn{4}{|l|}{ Museo de mujeres artistas mexicanas } \\
\hline Kvinnemuseet & $x$ & $x$ & $x$ \\
\hline \multicolumn{4}{|l|}{ Museum Anna Nordlander } \\
\hline The South Vietnam Women's Museum & $x$ & $x$ & $x$ \\
\hline Vietnam Women Museum & $x$ & $x$ & $x$ \\
\hline
\end{tabular}


A partir de los resultados encontrados en el estudio podemos concluir que los museos de mujeres son escasos, cuatro en España, de aproximadamente 1600 registrados en el Directorio de Museos y Colecciones dependiente del Ministerio de Cultura y Deporte, y tienen poca presencia en comunidades virtuales como TripAdvisor, de los cuarenta y ocho registrados por el Ministerio de Cultura y Deporta, sólo veinte tienen presencia en TripAdvisor. La escasa presencia en esta comunidad virtual limita la visibilidad y en consecuencia, las posibilidades de atraer público. Otra consecuencia de la falta de presencia en dicha comunidad es que el visitante no tiene información que le ayude a reducir la incertidumbre en la elección de la visita a un museo de mujeres (Bickart y Schindler, 2001).

En cuanto al volumen de eWOM, los resultados muestran la importancia de potenciar entre los visitantes y usuarios su participación e interacción en las comunidades virtuales compartiendo sus experiencias para que actúen como un motivador en la toma de decisiones de la visita de los públicos. Por otro lado, los hallazgos en los contenidos de eWOM publicados por los visitantes muestran que los comentarios de sus experiencias son, en general, positivos en un $85 \%$ de los casos estudiados, como se muestra en la Tabla 5.

\section{Conclusiones}

Los museos de mujeres se encuentran en un mercado altamente competitivo, no solamente con otros museos y organizaciones artísticas, sino también con distintos proveedores de ocio. En el caso de los museos de mujeres cuentan con una ventaja competitiva por el elevado interés que despierta el papel de la mujer en la sociedad actual.

Al igual que en otros ámbitos, las instituciones museísticas han sufrido profundas transfor- maciones para adaptarse a las necesidades de la sociedad. Desde los años 50 con la creación de los primeros museos de mujeres hasta nuestros días, éstos han evolucionado no solamente en la forma de exponer su contenido cultural, sino también en la manera de comunicarse; principalmente desde el auge y difusión de internet y las nuevas tecnologías que han generado importantes cambios en el comportamiento y en los procesos de comunicación de las personas (Lee, Park y Han, 2008) convirtiéndose en un fenómeno social de gran importancia en la sociedad. Mediante este medio, el influyente «de boca en boca» tradicional se ha transformado en el «de boca en boca» electrónico (eWOM), generando un gran impacto a la hora de tomar decisiones en el consumo cultural. Actualmente los consumidores se comunican a través de los nuevos entornos digitales, participando de forma activa mediante la generación de contenido sobre sus experiencias, opiniones y valoraciones influyendo en otros consumidores.

No existen estudios que confirmen por qué algunas opiniones son más influyentes que otras (Lim, Chung y Hew, 2011) ni cuantas opiniones son necesarias para influir en otros consumidores a la hora de tomar su decisión de consumo. La investigación académica puede aportar un modelo que explique qué opiniones son más influyentes, analizando diversos factores, así como establecer cuál es el número mínimo de opiniones necesario para considerarse un factor de influencia.

Los resultados hallados en la investigación confirman la importancia de analizar el eWOM en el ámbito turístico en general y en el caso de los museos de mujeres en particular, ya que es una de las fuentes más utilizadas por los turistas (Dey y Sarma, 2009; Gretzel y Yoo, 2008) de cara a planificar su tiempo de ocio en el destino turístico elegido. Es empleada para reducir el riesgo y la incertidumbre antes de tomar una decisión (Litvin, 
Goldsmith y Pan, 2008) como consecuencia de la intangibilidad y la imposibilidad de probar el producto antes del consumo (Lewis y Chambers, 2000). El eWOM permite que la información generada por los visitantes de un museo de mujeres dure en el tiempo, aumenta la credibilidad de la información en comunidades como TripAdvisor al ir acompañada de fotografías o videos de los visitantes, ayuda a la rápida información y facilita la interacción bidireccional entre los usuarios ya que pueden formularse preguntas para aclarar dudas tras la lectura de la información facilitada por un visitante.

Los museos no pueden controlar directamente el efecto del eWOM, pero sí pueden conocer cómo funciona esta herramienta de comunicación digital para aprovechar las ventajas y beneficios que ofrece, para así diseñar campañas de comunicación que les ayuden a generar un eWON positivo (Kozinets et al., 2010) hacia sus instituciones. Este trabajo puede ser de utilidad para los museos y en concreto para los museos de mujeres, ayudándoles a comprender la importancia de conocer a su visitante mediante la identificación del valor que este percibe de las opiniones y comentarios online realizados por visitantes anteriores y la influencia que pueden tener en su decisión de acudir al museo. Mediante el análisis del eWOM los museos de mujeres pueden identificar los factores que influyen de manera positiva en la decisión de visitar el museo, sean estos factores físicos del museo o emocionales del visitante. Esta información ayudará a los museos de mujeres a potenciar su reputación y conocimiento para aumentar el número de visitantes y con ello mejorar su posicionamiento. Además, el eWOM puede ayudar a los museos de mujeres a darse a conocer y construir una imagen de marca mediante la generación de contactos y experiencias de los visitantes, contribuyendo con ello al aumento de visitas y en consecuencia al aumento de sus ingresos. También les puede ayudar a captar ideas que los visitantes exponen para mejorar sus instalaciones, exposiciones y servicios ofertados.

Las principales limitaciones que plantea el presente trabajo son dos. Por un lado, solo se ha estudiado una comunidad virtual, TripAdvisor y, por otro lado, la muestra de museos de mujeres se ha limitado a los datos publicados por el Ministerio de Educación, Cultura y Deporte. Estas limitaciones orientaran otras líneas futuras de investigación, donde se planteará ampliar la muestra de museos de mujeres e incluir otras comunidades virtuales.

El desarrollo de este trabajo se centra principalmente, en el análisis del eWOM desde la perspectiva de los visitantes. Como línea futura de investigación se plantea profundizar en este análisis desde la perspectiva de los museos de mujeres. Analizar cómo estas instituciones pueden beneficiarse de esta comunicación para mejorar sus ingresos, sus instalaciones, servicios y contenido expuesto adaptado a la demanda de sus visitantes, así como aumentar la participación de los visitantes en la comunicación eWOM para favorecer la reputación e imagen de los museos de mujeres. 


\section{Bibliografía}

Ahluwalia, R. (2002). How Prevalent Is the Negativity Effect in Consumer Environments? Journal of Consumer Research, 29(2), 270-279. https://doi.org/10.1086/341576

Arndt, J. (1967). Role of Product-Related Conversations in the Diffusion of a New Product. Journal of Marketing Research, IV(August), 291-295.

Babić, A., Sotgiu, F, Valck, K. De, \& Bijmolt, T. H. A. (2015). The Effect of Electronic Word of Mouth on Sales : A MetaAnalytic Review of Platform, Product, and Metric Factors. American Marketing Association, 53(3), 297-318.

Bakos, Y., \& Dellarocas, C. (2011). Cooperation without enforcement? A comparative analysis of litigation and online reputation as quality assurance mechanisms. Management Science, 57(11), 1944-1962. https://doi.org/10.1287/ mnsc. 1110.1390

Balmer, J. M. T., \& Gray, E. R. (2003). Corporate brands: what are they? What of them? European Journal of Marketing, 37(7/8), 972-997. https://doi.org/10.1108/03090560310 477627

Banerjee, S., \& Chua, A. Y. (2016). In search of patterns among travellers' hotel ratings in TripAdvisor. Tourism Management, 53, 125-131.

Benito, V. L., \& Molina, N. L. (2010). Una panorámica de los museos de mujeres en el mundo. HerE - Mus. Heritage $\mathcal{E}$ Museography, (03), 12-18.

Bickart, B., \& Schindler, R. M. (2001). Internet Forums As Influential Sources of Consumer Information. Journal of Interactive Marketing, 15(3), 31-40.

Bowman, D., \& Narayandas, D. (2001). Managing customer-initiated contacts with manufacturers: The impact on share of category requirements and word-of-mouth behavior. Journal of Marketing Research, 38(3), 281-297. https:// doi.org/10.1509/jmkr.38.3.281.18863

Cáceres, M. J. M. (2010). El Museo Etnológico de la Mujer Gitana: un espacio social para hacer visible lo invisible. HerEMus. Heritage \& Museography, (03), 50-54.

Centro Pompidou (2019). Mujeres artistas en las colecciones del Museoa Nacional de Arte Moderno. Recuperado de https://www.centrepompidou.fr/cpv/agenda/event. action?param.id=FR_R28334c6bee53a6e8533a4449ae5 5394 e\&param.idSource $=F R \_E-d c 64 d 3 e e 3 d 83 d$ 6d265be583d2a67d238
Chen, Y., Wang, Q., \& Xie, J. (2011). Online Social Interactions: A Natural Experiment on Word of Mouth Versus Observational Learning. Journal of Marketing Research, 48(2), 238-254. https://doi.org/10.1055/s-0038-1626340

Cothrel, J. P. (2000). Measuring the success of an online community. Strategy E Leadership, 28(2), 17-21.

De Carvalho, I. M. D. M., \& Vaquinhas, M. (2015). Museus das mulheres na actualidade: Criação, objectivos e o contributo da história. RITUR-Revista Iberoamericana de Turismo, 5, 5-26.

Del Río-Castro, J. N. (2011). La web 2.0 en museos online de arte contemporáneo. Vivat Academia, (117), 1310-1327.

Dey, B., \& Sarma, M. K. (2010). Information source usage among motive-based segments of travelers to newly emerging tourist destinations. Tourism management, 31(3), 341-344.

Doh, S. J., \& Hwang, J. S. (2009). How consumers evaluate eWOM (electronic word-of-mouth) messages. Cyberpsychology and Behavior, 12(2), 193-197. https://doi. org/10.1089/cpb.2008.0109

Duygun, A., Menteş, S. A., \& Kubaş, A. (2014). The Impacts of Complaint Satisfaction on Corporate Reputation: A Study on Banking Sector. International Journal of Trade, Economics and Finance, 5(2), 159-160. https://doi.org/10.7763/ ijtef.2014.v5.361

East, R., Romaniuk, J., Chawdhary, R., \& Uncles, M. (2017). The Impact of Word of Mouth on Intention to Purchase Currently Used and other Brands. International Journal of Market Research, 59(3), 321-334. https://doi. org/10.2501/ijmr-2017-026

Engel, J. F., Kegerreis, R. J., \& Blackwell, R. D. (1969). Word-of-Mouth Communication by the Innovator. Journal ofMarketing,33(3), 15-19.https://doi.org/10.2307/1248475 Ferguson, M.; Piché, J., \& Walby, K. (2015). "Bridging or fostering social distance? An analysis of penal spectator comments on Canadian penal history museums." Crime, media, culture, 11(3), 357-374. http://dx.doi.org/10.1177/1 741659015596111

Filieri, R. \& McLeay, F. (2014). "E-WOM and accommodation: an analysis of the factors that influence travelers adoption of information from online reviews", Journal of Travel Research, 53 (1), 44-57. https://doi.org/10.1177/004728 7513481274

Floyd, K., Freling, R., Alhoqail, S., Cho, H. Y., \& Freling, T. (2014). How online product reviews affect retail sales: A 
meta-analysis. Journal of Retailing, 90(2), 217-232. https:// doi.org/10.1016/j.jretai.2014.04.004

Galarza Fernández, E., Castro-Martinez, A., \& Sosa Valcarcel, A. (2019). Medios sociales y feminismo en la construcción de capital social: la red estatal de comunicadoras en España. Anàlisi: quaderns de comunicació i cultura, 61, 0001-16.

Gavilan, D., Martínez-Navarro, G., \& Fernández-Lores, S. (2018). Comunicación en el sector turístico. Puntuaciones y comentarios de usuarios como variables estratégicas/Communication in the tourist sector. Scores and comments from users as strategic variables. Vivat Academia, (144), 77-94.

Gretzel, U., \& Yoo, K. H. (2008). Use and impact of online travel reviews. Information and communication technologies in tourism, 35-46.

Godes, D., \& Mayzlin, D. (2004). Using online conversations to study word-of-mouth communication. Marketing Science, 23(4), 545-560. https://doi.org/10.1287/mksc. 1040.0071

Goldsmith, R. E., \& Horowitz, D. (2006). Measuring Motivations for Online Opinion Seeking. Journal of Interactive Advertising, 6(2), 3-14. https://doi.org/10.1080/15252019. 2006.10722114

Hausmann, A. (2012). The importance of word of mouth for museums: An analytical framework. International Journal of Arts Management, 14(3), 32-43.

Hennig-Thurau, T., Gwinner, K. P., Walsh, G., \& Gremler, D. D. (2004). Electronic word-of-mouth via consumeropinion platforms: What motivates consumers to articulate themselves on the Internet? Journal of Interactive Marketing, 18(1), 38-52. https://doi.org/10.1002/dir.10073

Hennig-Thurau, T., Malthouse, E. C., Friege, C., Gensler, S., Lobschat, L., Rangaswamy, A., \& Skiera, B. (2010). The impact of new media on customer relationships. Journal of Service Research, 13(3), 311-330. https://doi.org/10. $1177 / 1094670510375460$

Hennig-Thurau, T., \& Walsh, G. (2003). Electronic wordof-mouth: Motives for and consequences of reading customer articulations on the internet. International Journal of Electronic Commerce, 8(2), 51-74. https://doi.org/10.1080/1 0864415.2003 .11044293

Hidayat, A., Mahdaria, S., Linando, J. A., \& Ishak, A. (2017). the Impact of Perceived Quality and Corporate Reputation Towards Positive Word-of-Mouth: Trust As Moderating. Science International, 29(2), 395-398.
Ho, H. Y., \& Chang, C. P. H. (2010). Influence of message trust in online word-of-mouth on consumer behavior - By the example of food blog. ICEIE 2010 - 2010 International Conference on Electronics and Information Engineering, Proceedings, 1(Iceie), 395-399. https://doi.org/10.1109/ICEIE. 2010.5559850

Hong, S. Y., \& Yang, S. (2009). Effects of Reputation, Relational Satisfaction, and Customer - Company Identification on Positive Word-of-Mouth Intentions. Journal of Public Relations Research, 21(4), 381-403. https://doi.org/10.1080/ 10627260902966433

Hsu, C. L., Lin, J. C. C., \& Chiang, H. Sen. (2013). The effects of blogger recommendations on customers' online shopping intentions. Internet Research, 23(1), 69-88. https://doi.org/10.1108/10662241311295782

Hwang, H. \& Kim, K. (2015). «Social media as a tool for social movements: The effect of social media use and social capital on intention to participate in social movements». International Journal of Consumer Studies, 39 (5), 478488. https://doi.org/10.1111/ijcs.12221

ICOM. (2019). Definición de museo. Recuperado de http:// icom.museum/the-vision/museum-definition/

International Association of Women's Museums, IAWM (2019). Objetivos de la asociación internacional de museos de mujeres. Recuperado de https://iawm.international/ about-us-2/our-goals/

Ismagilova, E., Slade, E. L., Rana, N. P., \& Dwivedi, Yogesh K. (2019). The effect of electronic word of mouth communications on intention to buy : A meta- analysis . Information Systems Frontiers, 1-24. Retrieved from https://doi. org/10.1007/s10796-019-09924-y\%0AThe

Jeacle, I., \& Carter, C. (2011). In TripAdvisor we trust: Rankings, calculative regimes and abstract systems. Accounting, Organizations and Society, 36(4-5), 293-309.

Katz, E., \& Lazarsfeld, P. F. (1955). Personal influence. Glencoe: Free.

Kim, W. G., \& Park, S. A. (2017). Social media review rating versus traditional customer satisfaction: Which one has more incremental predictive power in explaining hotel performance? International Journal of Contemporary Hospitality Management, 29(2), 784-802.

Koo, D. M. (2016). Impact of tie strength and experience on the effectiveness of online service recommendations. 
Electronic Commerce Research and Applications, 15(December), 38-51. https://doi.org/10.1016/j.elerap. 2015.12.002

Kotler, N., \& Kotler, P. (2000). "Can museums be all things to all people?: Missions, goals, and marketing's role." Museum management and curatorship 18(3), 271-287. https://doi. org/10.1080/09647770000301803

Kozinets, R.V. (2010) Networked narratives: understanding word-of-mouth marketing in online communities. Journal of Marketing, 74 (2), 71-89.

Lee, J., Park, D. H., \& Han, I. (2008). The effect of negative online consumer reviews on product attitude: An information processing view. Electronic Commerce Research and Applications, 7(3), 341-352. https://doi.org/10.1016/j. elerap.2007.05.004

Lee, M. K. O., Shi, N., Cheung, C. M. K., Lim, K. H., \& Sia, C. L. (2011). Consumer's decision to shop online: The moderating role of positive informational social influence. Information and Management, 48(6), 185-191. https://doi. org/10.1016/j.im.2010.08.005

Lewis, R., \& Chambers, R. (2000). Marketing leadership in hospitality: Foundations and practices. NY: John Wiley $\&$ Sons.

Lim, SCR, Cheung, WS \& Hew, KF (2011). Pensamiento crítico en la discusión asíncrona en línea: una investigación de las técnicas de facilitación de estudiantes. Nuevos horizontes en la educación, 59 (1), 52-65.

Litvin, S. W., Goldsmith, R. E., \& Pan, B. (2008). Electronic word-of-mouth in hospitality and tourism management. Tourism management, 29(3), 458-468.

Liu, X. (2019). Analyzing the impact of user-generated content on B2B Firms' stock performance: Big data analysis with machine learning methods. Industrial Marketing Management, (July 2018), 1-10. https://doi.org/10.1016/j.indmarman.2019.02.021

Liu, Y. (2006). Word of Mouth for Movies: Its Dynamics and Impact on Box Office Revenue. Journal of Marketing, 70, 74-89. https://doi.org/10.1016/j.dss.2016.11.002

López, M., \& Sicilia, M. (2014). Determinants of E-WOM influence: The role of consumers' internet experience. Journal of Theoretical and Applied Electronic Commerce Research, 9(1), 28-43. https://doi.org/10.4067/S0718-18762014 000100004

Ministerio de Cultura y Deporte de España (2019) Recuperado de http://www.culturaydeporte.gob.es/cultura/areas/ museos/mc/ceres/catalogos/catalogos-tematicos/patrimo- niofemenino/presentacion/enlaces-a-museos-de-mujeres. html

Mkono, M., \& Tribe, J. (2016). Beyond reviewing: Uncovering the multiple roles of tourism social media users. Journal of Travel Research, 56(3), 287-298.

Museo del Prado (2019). Exposición actualidad Historia de dos pintoras: Sofonisba Anguissola y Lavinia Fontana. Recuperado de https://www.museodelprado.es/actualidad/exposicion/historia-de-dos-pintoras-sofonisba-anguissolay/5f6c56c8-e8la-bf38-5f3f-9a2c2f5c60eb

Museo Nacional Centro de Arte Reina Sofía (2018). Exposición Dora García. Segunda Vez. Recuperado de https:// www.museoreinasofia.es/sites/default/files/dossier_dora_ garcia_para_web.pdf

Organización de Naciones Unidas (ONU), 2019. Cronograma: igualdad de género. Resumen del año 2018. Recuperado de https://interactive.unwomen.org/multimedia/timeline/ yearinreview/2018/es/index.html

Organización de Naciones Unidas Mujeres (2019) Cronograma https://interactive.unwomen.org/multimedia/timeline/womenunite/es/index.html\#/closing

Park, C., \& Lee, T. M. (2009). Information direction, website reputation and eWOM effect: A moderating role of product type. Journal of Business Research, 62(1), 61-67. https://doi.org/10.1016/j.jbusres.2007.11.017

Pearce, P. L., \& Wu, M. Y. (2018). Entertaining international tourists: An empirical study of an iconic site in China. Journal of Hospitality \& Tourism Research, 42(5), 772-792.

Reyes-Menendez, A., Saura, J. R., \& Martinez-Navalon, J. G. (2019). The Impact of e-WOM on Hotels Management Reputation: Exploring TripAdvisor Review Credibility with the ELM Model. IEEE Access, 7, 68868-68877. https://doi. org/10.1109/ACCESS.2019.2919030

Robinson, J. \& Valor, C. (2013) El Word of Mouth: construcción de un modelo del proceso a partir de la revisión de la literatura. Revista Internacional de Investigación en Comunicación aDResearch ESIC, 8(8), 8-25.

Saleem, A., \& Ellahi, A. (2017). Influence of electronic word of mouth on purchase intention of fashion products in social networking websites. Pakistan Journal of Commerce and Social Sciences (PJCSS), 11(2), 597-622.

Schönweger, A. (2010). Network Woman in Museum. Museos de la mujer se conectan entre sí. HerEMus. Heritage $\mathcal{E}$ Museography, (03), 55-66. 
Silverman, G. (2001). The Secrets of Word-of-Mouth Marketing (2a). https://doi.org/10.1109/OPTIM.2008.4602378

Sparks, B. A., Perkins, H. E., \& Buckley, R. (2013). Online travel reviews as persuasive communication: The effects of content type, source, and certification logos on consumer behavior. Tourism Management, 39, 1-9.

Tata, S. V., Prashar, S., \& Gupta, S. (2019). An examination of the role of review valence and review source in varying consumption contexts on purchase decision. Journal of Retailing and Consumer Services, (January), 1-10. https://doi. org/10.1016/j.jretconser.2019.01.003

TripAdvisor (2019). Clasificación a través de los Traveller's Choice. Recuperado de https://conocedores.com/mejoresmuseos-mundo-19093

Tsao, W. C., \& Hsieh, M. T. (2015). eWOM persuasiveness: do eWOM platforms and product type matter? Electronic Commerce Research, 15(4), 509-541. https://doi.org/10. 1007/s10660-015-9198-z

Williams, M., Buttle, F., \& Biggemann, S. (2012). Relating Word-of-Mouth to Corporate Reputation. Public Communication Review, 2(2), 3-16. https://doi.org/10.5130/pcr.v2i2.2590
Xie, K. L., Zhang, Z., \& Zhang, Z. (2014). The business value of online consumer reviews and management response to hotel performance. International Journal of Hospitality Management, 43, 1-12.

Xun, J., \& Guo, B. (2017). Twitter as customer eWOM: an empirical study on their impact on firm financial performance. Internet Research, 27(5), 1014-1038. https:// doi.org/10.1108/qmr.2005.21608cag.001

Yoo, Y.; Lee, T. J., \& Lee, C. (2015). "Effect of health and wellness values on festival visit motivation", Asia Pacific Journal of Tourism Research, 20 (2), 236-263. https://doi. org/10.1080/10941665.2013.866970

You, Y., Vadakkepatt, G. G., \& Joshi, A. M. (2015). A Meta-Analysis of Electronic Word-of-Mouth Elasticity. Journal of Marketing, 79(March), 19-39.

Zhang, Z., Zhang, Z., \& Yang, Y. (2016). The power of expert identity: How websiterecognized expert reviews influence travelers' online rating behavior. Tourism Management, 55, 15-24. 